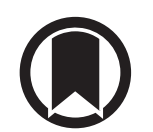

CrossMark

\title{
Chronic Airway Diseases Early Stratification (CADSET): a new ERS Clinical Research Collaboration
}

\author{
Alvar Agusti ${ }^{1,2}$, Rosa Faner², Gavin Donaldson ${ }^{3}$, Elise Heuvelin ${ }^{4}$, \\ Robab Breyer-Kohansal ${ }^{5}$, Erik Melén $\mathbb{1}^{6}$, Anke H. Maitland-van der Zee ${ }^{7,8}$, \\ Jørgen Vestbo ${ }^{9}$, James P. Allinson ${ }^{3}$, Lowie E.G.W. Vanfleteren (10) ${ }^{10}$, \\ Maarten van den Berge ${ }^{11}$, lan M. Adcock ${ }^{12}$, Lies Lahousse (1) ${ }^{13}$, \\ Guy Brusselle ${ }^{13}$ and Jadwiga A. Wedzicha ${ }^{3}$ on behalf of the CADSET \\ Clinical Research Collaboration ${ }^{14}$
}

Affiliations: ${ }^{1}$ Respiratory Institute, Hospital Clinic, IDIBAPS, University of Barcelona, Barcelona, Spain. ${ }^{2} \mathrm{CIBER}$ Enfermedades Respiratorias, Barcelona, Spain. ${ }^{3}$ Asthma and COPD Group, Airways Disease Section, Imperial College London, National Heart and Lung Institute, London, UK. "European Respiratory Society, Lausanne, Switzerland. ${ }^{5}$ Dept of Respiratory and Critical Care Medicine and Ludwig Boltzmann Institute for COPD and Respiratory Epidemiology, Otto Wagner Hospital, Vienna, Austria. 'Sachs' Children's Hospital and Institute of Environmental Medicine, Karolinska Institutet, Stockholm, Sweden. ${ }^{7}$ Dept of Respiratory Medicine, Amsterdam University Medical Centers, University of Amsterdam, Amsterdam, The Netherlands. ${ }^{8}$ Pediatric Respiratory Medicine, Emma Children's Hospital, Amsterdam University Medical Centers, University of Amsterdam, Amsterdam, The Netherlands. "Manchester University NHS Foundation Trust, Manchester Academic Health Science Centre, and Division of Infection, Immunity and Respiratory Medicine, University of Manchester, Manchester, UK. ${ }^{10}$ COPD Center, Institute of Medicine, Sahlgrenska University Hospital, University of Gothenburg, Gothenburg, Sweden. ${ }^{11}$ University of Groningen, University Medical Center Groningen, Groningen, The Netherlands. ${ }^{12}$ Cell and Molecular Biology Group, Airways Disease Section, Imperial College London, National Heart and Lung Institute, London, UK. ${ }^{13}$ Ghent University, Ghent, Belgium. ${ }^{14} \mathrm{~A}$ full list of the members of the CADSET Clinical Research Collaboration can be found at the end of this article.

Correspondence: Alvar Agusti, Respiratory Institute, Hospital Clinic. C/Villarroel 170, 08036 Barcelona, Spain. E-mail: aagustidaclinic.cat

@ERSpublications

The ERS launches a new Clinical Research Collaboration (CADSET) to study the determinants and implications of different lung function trajectories through life http://ow.ly/nf4E30nGsJG

Cite this article as: Agusti A, Faner R, Donaldson G, et al. Chronic Airway Diseases Early Stratification (CADSET): a new ERS Clinical Research Collaboration. Eur Respir J 2019; 53: 1900217 [https://doi.org/ 10.1183/13993003.00217-2019].

\section{Introduction}

A recent editorial in the European Respiratory Journal highlighted the strategic importance of the Clinical Research Collaborations (CRCs) launched in 2013 by the European Respiratory Society (ERS) [1]. These have the aim of 1) promoting the exchange of research ideas among clinicians and affiliated scientists in Europe and/or globally; 2) building an infrastructure for prospective clinical research; 3) securing additional funding through national and European Union funding streams; and 4) facilitating the planning, implementation, evaluation and publication of clinical and translational studies at pan-European level and beyond. So far, there are currently 17 ongoing CRCs that cover eight major respiratory disease domains (airway diseases, interstitial lung diseases, pulmonary vascular diseases, sleep and breathing disorders, respiratory critical care, paediatric respiratory diseases, respiratory infections and thoracic 
oncology), all of them linked to one or more ERS assemblies [2-12]. CADSET, an acronym that stands for "Chronic Airway Diseases Early Stratification", is the latest addition to the list of ongoing CRCs (www. ersnet.org/research/clinical-research-collaborations). This editorial presents the rationale, goals and research strategy for CADSET.

\section{Scientific rationale}

Asthma and chronic obstructive pulmonary disease (COPD) are the two most prevalent chronic respiratory diseases and neither has a curative therapy $[13,14]$. A third of the population will develop asthma at some point in their lives [13], and COPD affects $10 \%$ of the adult population, currently being the third leading cause of noncommunicable deaths globally [15]. Both asthma and COPD are complex and heterogeneous diseases, and their differential diagnosis is often not straightforward [16-20]. Indeed, it is suggested that asthma and COPD might actually represent a continuum of chronic airway diseases with shared clinical, functional, imaging and/or biological features [21]. However, asthma is still considered a disease of infancy or early adulthood, often related to some form of allergy [22], whereas COPD has been traditionally considered a disease of late adulthood, induced by smoking, and generally associated with an accelerated decline in lung function with age [23]. As a result, asthma is the most likely diagnosis that a child, adolescent or young adult will receive if they complain of respiratory symptoms, whereas a COPD diagnosis would be most likely in an older male smoker presenting with similar respiratory symptoms.

By contrast, recent evidence argues that there is an important role for abnormal lung development in utero or after birth in the pathogenesis of both asthma and COPD [24-30]. It is very likely that abnormal lung development and accelerated lung function decline (which also occurs in asthma) are associated with different biological mechanisms (i.e. endotypes), requiring different therapeutic interventions [18]. Of note, some children with impaired lung function early in life may exhibit "lung function catch-up" before adulthood (figure 1) [31-40], although the precise factors and mechanisms involved are currently unknown. A better understanding of the pathophysiology of this "catch-up" (or its absence) may prove extremely important in helping us to optimise lung growth, discern the reasons why particular individuals develop disease and, eventually, provide a novel therapeutic approach in adults with COPD [41, 42]. In any case, the available evidence indicates that to better understand the pathobiology of asthma and COPD, increase the specificity of their diagnosis and improve both the efficacy and safety of their treatment, we need to adopt a better understanding of the lung function trajectory of the patient throughout life (figure 1) [41].

\section{Working hypothesis}

From the aforementioned evidence, CADSET hypothesises that a multilevel (clinical, functional, imaging and molecular) profiling of well-characterised patients with chronic airways disease spanning the spectrum of asthma and COPD, which considers both peak lung function achieved in early adulthood and the rate of lung function decline, may lead to the identification of distinct mechanisms (endotypes) and appropriate biomarkers. These biomarkers may in turn inform a mechanism-based disease classification,

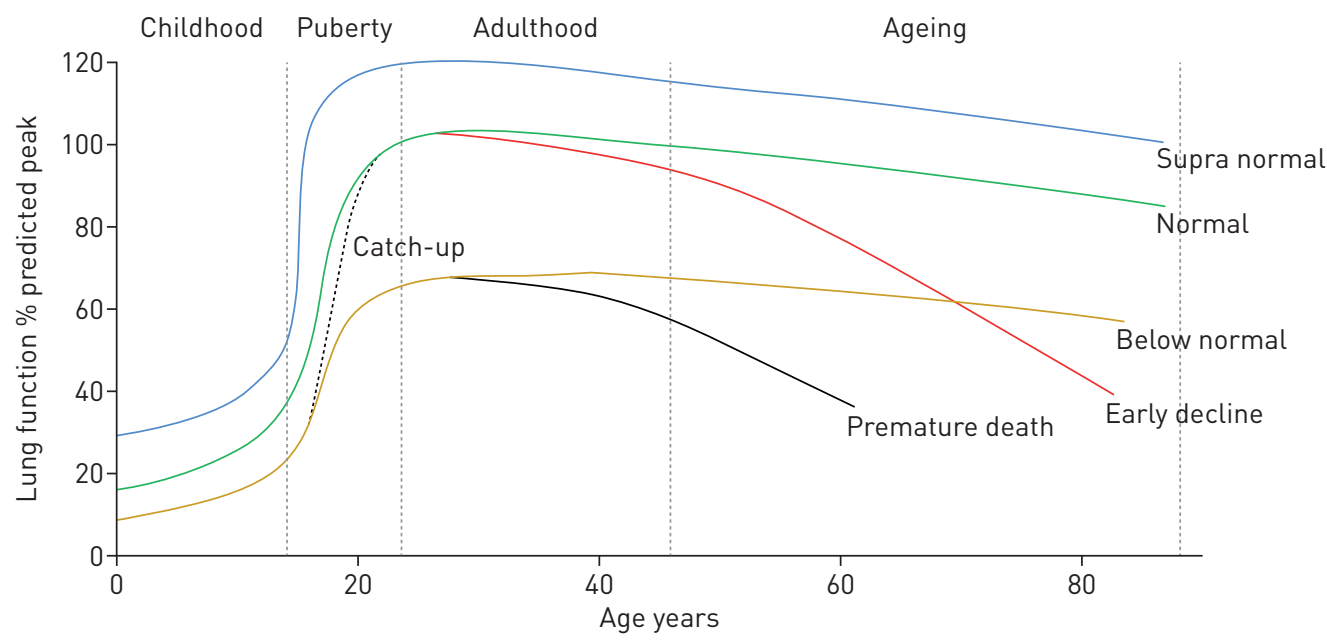

FIGURE 1 Schematic representation of different potential lung function trajectories through life. The term "catch-up" refers to the potential lung function recovery that may occur (for still unclear reasons) in some children. Reproduced and modified from [41] with permission. 
identify interventional targets and thus favour a more personalised treatment of patients with chronic airways diseases.

\section{Objectives}

The strategic objective of CADSET is to create a consortium in which investigators with access to population cohorts, birth cohorts and cohorts of patients with asthma or COPD can work together to better understand how lung function trajectories influence the clinical presentation of chronic airway diseases, asthma and COPD in particular. A second important strategic objective of CADSET is to help young investigators to develop their academic careers, as reflected by their management role as work package leaders in CADSET.

The specific objectives of CADSET are: 1) to provide a multicentre, multidisciplinary platform to investigate lung function trajectories on the clinical presentation of chronic airway diseases; 2) to create a registry of currently available cohorts (and associated metadata and/or existing biobanks) in order to launch joint meta-analyses and targeted laboratory studies; 3) to share expertise and resources, creating common research protocols in order to enable the comparison and integration of data across different types of cohorts; 4) to promote the exchange of research ideas among participants; 5) to find distinct biological mechanisms and endotypes, and associated biomarkers, that identify underlying different lung function trajectories in order to move COPD and asthma assessment and therapy towards a precision medicine framework; and 6) to gain eligibility for public and private funding.

\section{Research strategy}

Under the auspices of the ERS, CADSET is based on a collaborative partnership model between the different stakeholder groups, e.g. clinician researchers, scientific experts, funding partners and patient advisors, where the project proposals and results are openly discussed. The collaboration of all stakeholders aims to drive the research strategy of CADSET, which is organised on the basis of five different working groups (WGs), each of them with a clear focus.
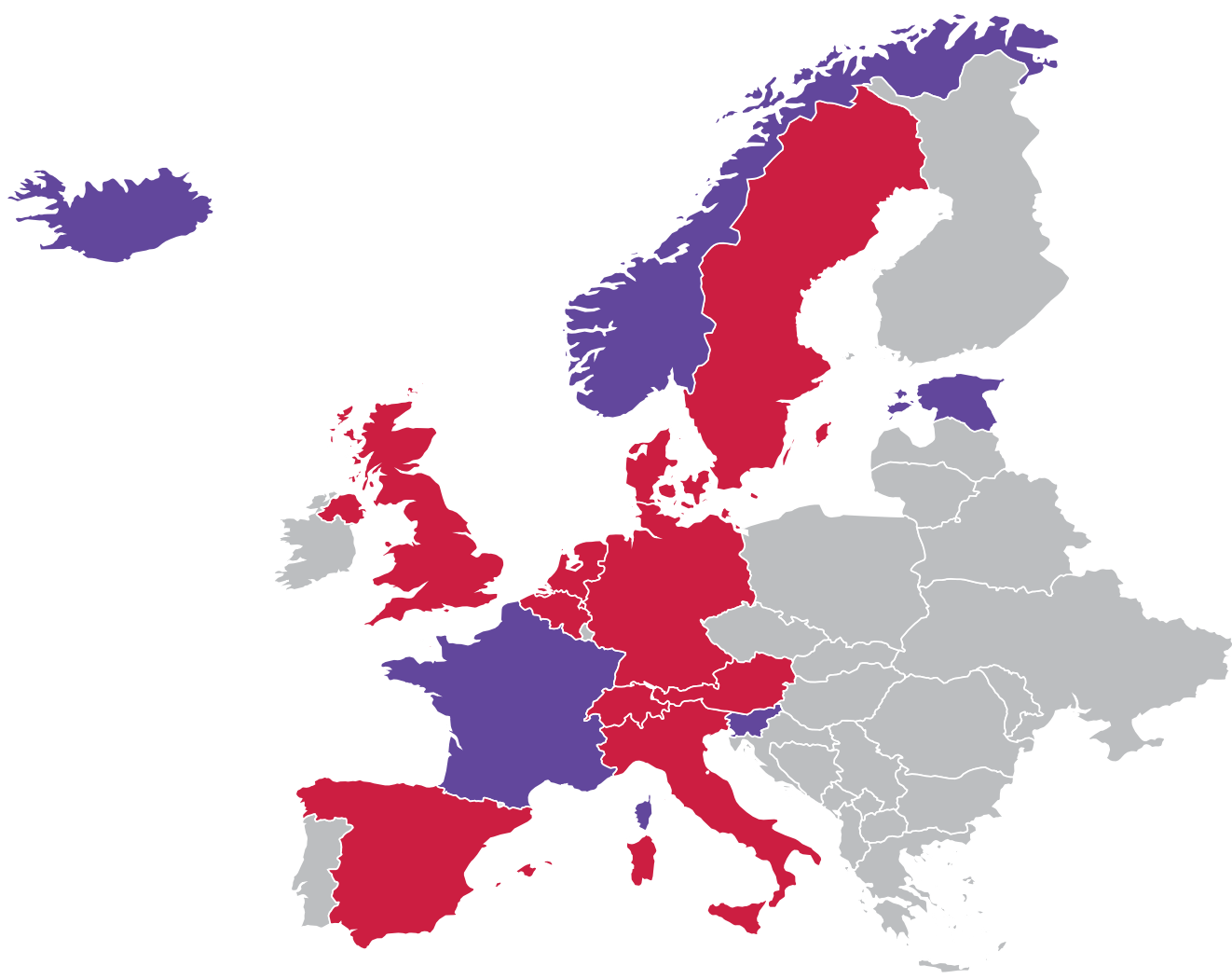

FIGURE 2 Cohorts and research groups currently participating in the Chronic Airway Diseases Early Stratification (CADSET) Clinical Research Collaboration. Red indicates countries with available cohorts and investigators, whereas purple indicates countries with available cohorts but not investigators (yet). 


\section{WG1: Registry}

WG1 aims to develop a registry of sharable consortium resources on existing cohorts (including environmental, clinical, physiological, 'omic and imaging data, and biobanked blood, sputum and tissue samples) and to collate and share best practices from research protocols and standard operating procedures. The aim of WG1 is not to physically centralise data and samples in a single place but to assess the feasibility of assembling such resources for further studies as identified in the other WGs, and for commissioned projects from external groups and industry partners. So far, 33 cohorts have been registered and are available in the CADSET registry (figure 2). Data and sample sharing will always require the respective cohort principal investigators and governance committee agreement and be undertaken according to the General Data Protection Regulation.

\section{WG2: From conception to age 25 years}

WG2 will investigate the phenotypes and endotypes of suboptimal peak lung function at early adulthood (aged $<25$ years). In particular, it will first investigate the existence of different early life lung function trajectories (e.g. linked to early bronchial obstruction ("asthma"), wheezing or symptoms due to other conditions, or suboptimal lung development) and the mechanisms underlying potential lung function catch-up [36, 41]. Stratified analyses according to sex will be performed (since females obtain their peak lung function at an earlier age than males [43]).

\section{WG3: From age 25 to 50 years}

WG3 will investigate the clinical and underlying biological heterogeneity and related biomarkers of lung function decline in early adulthood. This will focus particularly in relation to the presence and severity of emphysema, chronic mucus hypersecretion, concomitant asthma, eosinophilic inflammation, concomitant comorbidity and response to specific pharmacological treatment (precision medicine [44]). As in WG2, stratified analyses according to sex will be performed [43].

\section{WG4: From age 50 years onwards}

WG4 will integrate multilevel data such as genetics, genomics, methylation and proteomics on similar biological samples from well-defined populations with "asthma" or "COPD" in order to understand molecular phenotypes that may be common or distinct to chronic airway diseases in this age range. This will be linked to readily accessible physiological, blood or breath biomarkers.

\section{WG5: Understanding life-long lung function trajectories}

The long-term goal of WG5 is to integrate the age-related results of WG2, WG3 and WG4, so that the strategic goals of CADSET can be fulfilled. CADSET will collaborate with other established CRCs, such as SHARP (The Severe Heterogeneous Asthma Research collaboration, Patient-centred) [5] and the Global Lung Function Initiative (GLI) network [10].

\section{Support and participation}

As for the other CRCs, the ERS provides some economic support, mainly to allow the organisation of face-to-face meetings, such as the one held in Barcelona, Spain, in December 2018. Besides this, the ERS acts as an independent broker between industry and the academic researchers. It is hoped that several companies may be interested in supporting CADSET financially. Likewise, it is envisaged that CADSET investigators will be able to raise additional funds from national and international competitive calls.

If you want or can contribute to CADSET with a cohort that helps in achieving the objectives outlined here, please contact the WG members or the ERS through the CADSET webpage (www.ersnet.org/ research/cadset-chronic-airway-diseases-early-stratification).

Acknowledgements: We are grateful for the support from the ERS office in Lausanne, Switzerland, in logistics and organisation of CADSET.

Current members of the CADSET Clinical Research Collaboration are as follows. Co-chairs and WG5 co-leaders: Alvar Agusti and Jadwiga A. Wedzicha. WG1 co-leaders: Gavin Donaldson, Rosa Faner and Robab Breyer-Kohansal. WG2 co-leaders: Anke H. Maitland-van der Zee and Erik Melén. WG3 co-leaders: James P. Allinson, Lowie E.G.W. Vanfleteren and Jørgen Vestbo. WG4 co-leaders: Ian M. Adcock, Lies Lahousse and Maarten van den Berge. Additional CADSET members: Peter Alter, Ferran Barbe, Christopher E. Brightling, Marie-Kathrin Breyer, Otto C. Burghuber, Maribel Casas, Kian Fan Chung, Borja G. Cosío, Fatima Crispi, Jordi de Batlle, Jean-William Fitting, Judith Garcia, Jenny Hallberg, Sylvia Hartl, Deborah Jarvis, Alexander Mathioudakis, Laurent Nicod, Alberto Papi, Andrew Ritchie, Torben Sigsgaard, Peter J. Sterk, Anders Ullman, Kilian Vellvé, Claus Vogelmeier, Asa M. Wheelock, Craig E. Wheelock and Antonio Spanevello.

Conflict of interest: A. Agusti reports personal fees from AstraZeneca, Chiesi and Nuvaira, and grants and personal fees from Menarini and GSK, outside the submitted work. R. Faner reports support from GSK for an advisory board, and 
grants from GSK and Menarini, outside the submitted work. G. Donaldson has nothing to disclose. E. Heuvelin reports multi-sponsored study fees from Chiesi, Sanofi, Grifols, Novartis, Insmed, Zambon, TEVA, GlaxoSmithKline and CSL Behring, outside the submitted work. E. Heuvelin is an employee of the European Respiratory Society. R. Breyer-Kohansal reports grants and personal fees from AstraZeneca, Boehringer Ingelheim and Chiesi, personal fees from Novartis, and grants from GlaxoSmithKline, Menarini and Pfizer, during the conduct of the study. E. Melén has nothing to disclose. A.H. Maitland-van der Zee reports research grants from Boehringer Ingelheim, GSK and Novartis, and she participated in an advisory board from AstraZeneca, outside the submitted work. J. Vestbo reports personal fees for consultancy for COPD Phase 2 and 3 programme and payment for lectures including service in speaker bureaux, from GlaxoSmithKline, Chiesi Pharmaceuticals, Boehringer Ingelheim, Novartis and AstraZeneca, and an unconditional grant for biomarker research at Manchester University Hospital NHS Foundation Trust from Boehringer Ingelheim, outside the submitted work. J.P. Allinson has nothing to disclose. L.E.G.W. Vanfleteren reports grants and personal fees from AstraZeneca, personal fees from Pulmonx, Novartis, GSK and Menarini, grants from Fisher \& Paykel, and personal fees and non-financial support from Chiesi, outside the submitted work. M. van den Berge has nothing to disclose. I.M. Adcock has nothing to disclose. L. Lahousse reports grants from AstraZeneca and Chiesi (both awards), and expert consultation for Boehringer Ingelheim $\mathrm{GmbH}$ and Novartis, outside the submitted work. G. Brusselle reports personal fees for advisory boards and lecture fees from AstraZeneca, Boehringer Ingelheim, Chiesi, GlaxoSmithKline, Novartis and Teva, and personal fees for advisory boards from Sanofi, outside the submitted work. J.A. Wedzicha reports research grants to her institution from GSK and Johnson \& Johnson, research grants for early COPD cohorts from GSK, AstraZeneca, Boehringer Ingelheim, Novartis and Chiesi, and meeting expenses from Novartis, Boehringer Ingelheim, AstraZeneca and GSK, outside the submitted work.

Support statement: Funding was received from the European Respiratory Society.

\section{References}

1 Brightling C, Genton C, Bill W, et al. ERS Clinical Research Collaborations: underpinning research excellence. Eur Respir J 2018; 52: 1801534.

2 Bonsignore MR, Hedner J. The European Sleep Apnoea Database (ESADA) ERS Clinical Research Collaboration: past, present and future. Eur Respir J 2018; 52: 1801666.

3 Rusconi F, Fernandes RM, Pijnenburg MWH, et al. The Severe Paediatric Asthma Collaborative in Europe (SPACE) ERS Clinical Research Collaboration: enhancing participation of children with asthma in therapeutic trials of new biologics and receptor blockers. Eur Respir J 2018; 52: 1801665.

4 Aliberti S, Polverino E, Chalmers JD, et al. The European Multicentre Bronchiectasis Audit and Research Collaboration (EMBARC) ERS Clinical Research Collaboration. Eur Respir J 2018; 52: 1802074.

5 Djukanovic R, Adcock IM, Anderson G, et al. The Severe Heterogeneous Asthma Research collaboration, Patientcentred (SHARP) ERS Clinical Research Collaboration: a new dawn in asthma research. Eur Respir J 2018; 52: 1801671.

6 Cunningham S, Gilbert C, Schwerk N. The European research collaboration for Children's Interstitial Lung Disease (ChILDEU) ERS Clinical Research Collaboration. Eur Respir J 2018; 52: 1801855.

7 Sly PD, Hantos Z. The International Collaboration to Improve Respiratory Health in Children (INCIRCLE) ERS Clinical Research Collaboration. Eur Respir J 2018; 52: 1801867.

8 Martin-Loeches I, Torres A. The European Network for ICU-Related Respiratory Infections (ENIRRIs) ERS Clinical Research Collaboration. Eur Respir J 2019; 53: 1801972.

9 van Leth F, Brinkmann F, Cirillo DM, et al. The Tuberculosis Network European Trials group (TBnet) ERS Clinical Research Collaboration: addressing drug-resistant tuberculosis through European cooperation. Eur Respir J 2019; 53: 1802089 .

10 Hall GL, Stanojevic S. The Global Lung Function Initiative (GLI) Network ERS Clinical Research Collaboration: how international collaboration can shape clinical practice. Eur Respir J 2019; 53: 1802277.

11 Miravitlles M, Chorostowska-Wynimko J, Ferrarotti I, et al. The European Alpha-1 Research Collaboration (EARCO): a new ERS Clinical Research Collaboration to promote research in alpha-1 antitrypsin deficiency. Eur Respir J 2019; 53: 1900138.

12 Heunks L, Bellani G, Pham T, et al. The worldwide assessment of separation of patients from ventilatory assistance (WEAN SAFE) ERS Clinical Research Collaboration. Eur Respir J 2019; 53: 1802228

13 Global Initiative for Asthma (GINA). Global Strategy for Asthma Management and Prevention. 2018. Available from: https://ginasthma.org

14 Global Initiative for Chronic Obstructive Lung Disease (GOLD). Global Strategy for the Diagnosis, Management, and Prevention of Chronic Obstructive Pulmonary Disease. 2019. Available from: https://goldcopd.org

15 GBD 2017 Mortality Collaborators. Global, regional, and national age-sex-specific mortality and life expectancy, 1950-2017: a systematic analysis for the Global Burden of Disease Study 2017. Lancet 2018; 392: 1684-1735.

16 Pavord ID, Beasley R, Agusti A, et al. After asthma: redefining airways diseases. Lancet 2018; 391: 350-400.

17 Vanfleteren LE, Kocks JW, Stone IS, et al. Moving from the Oslerian paradigm to the post-genomic era: are asthma and COPD outdated terms? Thorax 2014; 69: 72-79.

18 Agusti A, Celli B, Faner R. What does endotyping mean for treatment in chronic obstructive pulmonary disease? Lancet 2017; 390: 980-987.

19 Agusti A, Bel E, Thomas M, et al. Treatable traits: toward precision medicine of chronic airway diseases. Eur Respir J 2016; 47: 410-419.

20 Agustí A, Bafadhel M, Beasley R, et al. Precision medicine in airway diseases: moving to clinical practice. Eur Respir J 2017; 50: 1701655

21 Bateman ED, Reddel HK, van Zyl-Smit RN, et al. The asthma-COPD overlap syndrome: towards a revised taxonomy of chronic airways diseases? Lancet Respir Med 2015; 3: 719-728.

22 Voraphani N, Stern DA, Wright AL, et al. Risk of current asthma among adult smokers with respiratory syncytial virus illnesses in early life. Am J Respir Crit Care Med 2014; 190: 392-398.

23 Fletcher C, Peto R. The natural history of chronic airflow obstruction. Br Med J 1977; 1: 1645-1648.

24 Lange P, Celli B, Agusti A, et al. Lung-function trajectories leading to chronic obstructive pulmonary disease. $N$ Engl J Med 2015; 373: 111-122. 


,
Belgrave DCM, Granell R, Turner SW, et al. Lung function trajectories from pre-school age to adulthood and their associations with early life factors: a retrospective analysis of three population-based birth cohort studies. Lancet Respir Med 2018; 6: 526-534.

33 Suresh S, O'Callaghan M, Sly PD, et al. Impact of childhood anthropometry trends on adult lung function. Chest 2015; 147: 1118-1126.

34 Mahmoud $\mathrm{O}$, Granell $\mathrm{R}$, Tilling $\mathrm{K}$, et al. Association of height growth in puberty with lung function. A longitudinal study. Am J Respir Crit Care Med 2018; 198: 1539-1548.

35 von Mutius E. Childhood origins of COPD. Lancet Respir Med 2018; 6: 482-483.

36 Melén E, Guerra S. Recent advances in understanding lung function development. F1000Res 2017; 6: 726.

37 Morgan WJ, Stern DA, Sherrill DL, et al. Outcome of asthma and wheezing in the first 6 years of life: follow-up through adolescence. Am J Respir Crit Care Med 2005; 172: 1253-1258.

38 Thunqvist P, Gustafsson PM, Schultz ES, et al. Lung function at 8 and 16 years after moderate-to-late preterm birth: a prospective cohort study. Pediatrics 2016; 137: e20152056.

39 Sonnenschein-van der Voort AM, Arends LR, de Jongste JC, et al. Preterm birth, infant weight gain, and childhood asthma risk: a meta-analysis of 147,000 European children. J Allergy Clin Immunol 2014; 133: 1317-1329.

40 Simpson SJ, Turkovic L, Wilson AC, et al. Lung function trajectories throughout childhood in survivors of very preterm birth: a longitudinal cohort study. Lancet Child Adolesc Health 2018; 2: 350-359.

41 Agusti A, Faner R. Lung function trajectories in health and disease. Lancet Respir Med 2019; in press [https://doi. org/10.1016/S2213-2600(18)30529-0].

42 Allinson JP, Hardy R, Donaldson GC, et al. Childhood exposures, asthma, smoking, interactions, and the catch-up hypothesis. Ann Am Thorac Soc 2018; 15: 1241-1242.

43 Kohansal R, Martinez-Camblor P, Agusti A, et al. The natural history of chronic airflow obstruction revisited: an analysis of the Framingham offspring cohort. Am J Respir Crit Care Med 2009; 180: 3-10.

44 Jameson JL, Longo DL. Precision medicine - personalized, problematic, and promising. N Engl J Med 2015; 372: 2229-2234. 